\title{
LOJASIEWICZ'S SUPPORT AND DOUBLY STOCHASTIC MEASURES
}

\author{
P. MIKUSIŃSKI, H. SHERWOOD, AND M. D. TAYLOR
}

(Communicated by R. Daniel Mauldin)

\begin{abstract}
We consider an alternative to the usual notion of the support of a measure which seems more suitable for the study of doubly stochastic measures.
\end{abstract}

\section{INTRODUCTION}

By a doubly stochastic measure (dsm) we mean a probability measure $\mu$ on the unit square $I^{2}(I=[0,1])$ such that

$$
\mu(A \times I)=\mu(I \times A)=\lambda(A)
$$

for every Borel set $A \subseteq I$; $\lambda$ is the Lebesgue measure.

The set of all dsm's (denoted by DSM) is clearly a convex set. Extremal dsm's (i.e. those which cannot be expressed as a convex combination of two distinct elements of DSM) are of special interest. We are concerned with the following question: Where is the mass of an extremal dsm concentrated? J. Lindenstrauss [2] shows that extremal dsm's are singular with respect to the Lebesgue measure on $I^{2}$. On the other hand, V. Losert [3] gives an example of an extremal dsm whose support is the whole of $I^{2}$. In this note we would like to discuss a different notion of support of a dsm which seems to describe better the set where the mass is concentrated. The concept of Lojasiewicz's support has been introduced in the theory of generalized functions [1]. We will show that the Lojasiewicz's support has been introduced in the theory of generalized functions [1]. We will show that the Lojasiewicz support has several nice features so that in some respect it is the "natural" way to define the support of a dsm. First, the Lojasiewicz support of an extremal dsm has Lebesgue measure zero; this dovetails nicely with Lindenstrauss's result. Second, one can use the general and ordinary derivatives of a measure [7] to define notions of support in a manner analogous to the definition of the Lojasiewicz support, and for dsm's, all three of these definitions are equivalent. Third, the convex

Received by the editors September 18, 1989 and, in revised form, October 13, 1989.

1980 Mathematics Subject Classification (1985 Revision). Primary 28A99; Secondary 28A35, $60 \mathrm{~A} 10$.

Key words and phrases. Doubly stochastic measure, generalized function, support of measure. 
sum of a sequence of probability measures on $I^{2}$ having Lojasiewicz supports whose Lebesgue measures are zero has the property that its Lojasiewicz support also has Lebesgue measure zero. This is not true for the standard definition of support.

Denote by $\mathscr{D}$ the space of all infinitely differentiable functions with compact support in $\mathbf{R}^{N}$, and by $\mathscr{D}^{\prime}$ the space of all Schwartz distributions on $\mathbf{R}^{N}$. If $f \in \mathscr{D}^{\prime}$ and $\varphi \in \mathscr{D}$, then we will use the notation

$$
(f, \varphi)=\int_{\mathbf{R}^{N}} f(x) \varphi(x) d x
$$

even if the integral does not exist in the usual sense.

Definition 1. Let $f \in \mathscr{D}^{\prime}$. We say that $f$ has a value $\beta$ at $x_{0} \in \mathbf{R}^{N}$, denoted by $f\left(x_{0}\right)=\beta$, if for every $\varphi \in \mathscr{D}, \varphi \geq 0, \int_{\mathbf{R}^{N}} \varphi(x) d x=1$, and for every $\alpha_{n} \rightarrow \infty\left(\alpha_{n}>0\right)$ we have

$$
\lim _{n \rightarrow \infty}\left(\alpha_{n}\right)^{N} \int_{\mathbf{R}^{N}} f(x) \varphi\left(\alpha_{n}\left(x-x_{0}\right)\right) d x=\beta .
$$

The value of a distribution can be also defined using the distributional convergence:

$$
f\left(x_{0}\right)=\beta \quad \text { if } \lim _{|\gamma| \rightarrow 0} f\left(x_{0}+\gamma x\right)=\beta \text { in } \mathscr{D}^{\prime} .
$$

If $f$ is a continuous function, then the limit (1) equals $f\left(x_{0}\right)$. The value of a distribution need not exist. For example, the Dirac delta distribution does not have a value at 0 ; it has value 0 at any other point.

The concept of the value of a distribution at a point has been introduced by S. Lojasiewicz in $[4,5]$ (see also [6, 8]).

Definition 2. The Lojasiewicz support of a distribution $f, \mathrm{~L}$-supp $f$, is the complement of the set of all points where $f$ has value 0 .

It can be shown that the closure of the Lojasiewicz support of a distribution is the support in the sense of Schwartz.

Every locally finite Borel measure $\mu$ on $\mathbf{R}^{N}$ can be identified with a distribution $f_{\mu}$ by

$$
\left(f_{\mu}, \varphi\right)=\int_{\mathbf{R}^{N}} \varphi d \mu
$$

In view of our notation it makes sense to denote it by $d \mu / d \lambda$ :

$$
\int_{\mathbf{R}^{N}} \varphi d \mu=\int_{\mathbf{R}^{N}} \varphi \frac{d \mu}{d \lambda} d \lambda .
$$

Since every dsm is a finite Borel measure it has a well-defined Lojasiewicz support.

Let $E_{1}, E_{2}, \ldots \subset \mathbf{R}^{N}, x \in \mathbf{R}^{N}$. We say that $\left\{E_{n}\right\}$ converges to $x$, and write $E_{n} \rightarrow x$, if $d\left(E_{n}\right) \rightarrow 0$ and $x \in \cap E_{n}$; where $d(E)=\sup \{\|x-y\|: x, y \in E\}$ and $\|\cdot\|$ denotes the Euclidean norm in $\mathbf{R}^{N}$. For measurable $E \in \mathbf{R}^{N}$ define

$$
r(E)=\sup \{\lambda(E) / \lambda(J): J \text { is a cube containing } E\} .
$$


A sequence of measurable sets $E_{n} \subset \mathbf{R}^{N}$ is called regular if there exists $\varepsilon>0$ such that $r\left(E_{n}\right)>\varepsilon$ for all $n \in \mathbf{N}$.

Definition 3 (Saks [7]). Let $\mu$ be a measure on $\mathbf{R}^{N}$. By the general derivative of $\mu$ at $x \in \mathbf{R}^{N}$, denoted by $D \mu(x)$, we mean the limit

$$
D \mu(x)=\lim _{n \rightarrow \infty} \mu\left(E_{n}\right) / \lambda\left(E_{n}\right),
$$

if it exists and is the same for any regular sequence of closed sets $E_{n} \in \mathbf{R}^{N}$ convergent to $x$.

By the ordinary derivative of $\mu$ at $x \in \mathbf{R}^{N}$, denoted by $\mu^{\prime}(x)$, we mean the limit

$$
\mu^{\prime}(x)=\lim _{n \rightarrow \infty} \mu\left(E_{n}\right) / \lambda\left(E_{n}\right),
$$

if it exists and is the same for any regular sequence of intervals $E_{n} \in \mathbf{R}^{N}$ convergent to $x$.

Theorem 4. Let $\mu$ be a locally finite Borel measure on $\mathbf{R}^{2}$ and let $p \in \mathbf{R}^{2}$. The following conditions are equivalent:

(a) $D \mu(p)=0$,

(b) $\mu^{\prime}(p)=0$,

(c) $\frac{d \mu}{d \lambda}(p)=0$.

Proof. (a) $\Rightarrow($ b) is obvious.

(b) $\Rightarrow$ (c). Suppose first $p=0$. Let $\varphi \in \mathscr{D}\left(\mathbf{R}^{N}\right), \int \varphi=1, \alpha_{1}, \alpha_{2}, \ldots>0$, $\alpha_{n} \rightarrow \infty$. Let $E$ be an interval in $\mathbf{R}^{N}$ such that $0 \in E$ and

$$
-c \chi_{E} \leq \varphi \leq c \chi_{E}
$$

where $c$ is a positive constant. Define $E_{n}=\left(1 / \alpha_{n}\right) E$. Then $E_{n} \rightarrow 0$. For all $x \in \mathbf{R}^{N}$ and all $n \in \mathbf{N}$, we have

$$
-c \chi_{E_{n}}(x) \leq \varphi\left(\alpha_{n} x\right) \leq c \chi_{E_{n}}(x)
$$

and

$$
-c\left(\alpha_{n}\right)^{N} \chi_{E_{n}}(x) \leq\left(\alpha_{n}\right)^{N} \varphi\left(\alpha_{n} x\right) \leq c\left(\alpha_{n}\right)^{N} \chi_{E_{n}}(x) .
$$

Denote $\varphi_{n}(x)=\left(\alpha_{n}\right)^{N} \varphi\left(\alpha_{n} x\right)$. Then

$$
-c\left(\alpha_{n}\right)^{N} \mu\left(E_{n}\right) \leq \int \varphi_{n} d \mu \leq c\left(\alpha_{n}\right)^{N} \mu\left(E_{n}\right),
$$

Since $\lambda\left(E_{n}\right)=\left(\alpha_{n}\right)^{-N} \lambda(E)$, we have

$$
-c \lambda(E) \frac{\mu\left(E_{n}\right)}{\lambda\left(E_{n}\right)} \leq \int \varphi_{n} d \mu \leq c \lambda(E) \frac{\mu\left(E_{n}\right)}{\lambda\left(E_{n}\right)} .
$$

Since

$$
\frac{\mu\left(E_{n}\right)}{\lambda\left(E_{n}\right)} \rightarrow 0
$$


we have

$$
\int \varphi_{n} d \mu \rightarrow 0
$$

which proves that

$$
\frac{d \mu}{d \lambda}(0)=0
$$

If $p \neq 0$, then consider the measure $\nu(A)=\mu(A-p)$.

(c) $\Rightarrow($ a) . Suppose

$$
p=0 \text { and } \frac{d \mu}{d \lambda}(0)=0
$$

First we will show that

$$
\lim _{n \rightarrow \infty} \frac{\mu\left(C_{n}\right)}{\lambda\left(C_{n}\right)}=0
$$

where $\left\{C_{n}\right\}$ is a sequence of cubes in $\mathbf{R}^{N}$. convergent to 0 . Denote

$$
\left\|C_{n}\right\|=\max _{x \in C_{n}}\|x\| \text {. }
$$

Then $\|x\| /\left\|C_{n}\right\| \leq 1$ whenever $x \in C_{n}$. Let $\varphi \in \mathscr{D}, \varphi \geq 0$, and $\varphi(x)=1$ for all $\|x\| \leq 1$. Then

and hence

$$
\chi_{C_{n}} \leq \varphi\left(x /\left\|C_{n}\right\|\right)
$$

$$
\mu\left(C_{n}\right) \leq \int \varphi\left(x /\left\|C_{n}\right\|\right) d \mu=\int \varphi d \lambda\left\|C_{n}\right\|^{N} \int \frac{\varphi\left(x /\left\|C_{n}\right\|\right)}{\int \varphi d \lambda\left\|C_{n}\right\|^{N}} d \mu .
$$

Consequently

$$
\frac{\mu\left(C_{n}\right)}{\lambda\left(C_{n}\right)} \leq \int \varphi d \lambda \frac{\left\|C_{n}\right\|^{N}}{\lambda\left(C_{n}\right)} \int \frac{\varphi\left(x /\left\|C_{n}\right\|\right)}{\int \varphi d \lambda\left\|C_{n}\right\|^{N}} d \mu .
$$

Since $\frac{d \mu}{d \lambda}(0)=0$, we have

$$
\lim _{n \rightarrow \infty} \int \frac{\varphi\left(x /\left\|C_{n}\right\|\right)}{\int \varphi d \lambda\left\|C_{n}\right\|^{N}} d \mu=0
$$

This implies

$$
\lim _{n \rightarrow \infty} \frac{\mu\left(C_{n}\right)}{\lambda\left(C_{n}\right)}=0
$$

because the sequence $\left\{\left\|C_{n}\right\|^{N} / \lambda\left(C_{n}\right)\right\}$ is bounded.

If $\left\{E_{n}\right\}$ is an arbitrary regular sequence of closed subsets of $\mathbf{R}^{N}$ convergent to 0 , then there is a sequence of cubes $C_{n}$ convergent to 0 and containing $E_{n}$ such that

$$
\frac{\lambda\left(C_{n}\right)}{\lambda\left(E_{n}\right)}<\theta
$$

for all $n \in \mathbf{N}$, such cubes exist because $\left\{E_{n}\right\}$ is regular. Now

$$
\frac{\mu\left(E_{n}\right)}{\lambda\left(E_{n}\right)} \leq \frac{\mu\left(C_{n}\right)}{\lambda\left(E_{n}\right)}=\frac{\mu\left(C_{n}\right)}{\lambda\left(C_{n}\right)} \frac{\lambda\left(C_{n}\right)}{\lambda\left(E_{n}\right)}<\theta \frac{\mu\left(C_{n}\right)}{\lambda\left(C_{n}\right)} \rightarrow 0,
$$


in view of the first part of this proof. Therefore $D \mu(0)=0$. Generalization to arbitrary $p$ is easy.

Theorem 5. The Lojasiewicz support of a locally finite Borel measure on $\mathbf{R}^{2}$ is a Borel set.

Proof. Let $\phi \in \mathscr{D}\left(\mathbf{R}^{2}\right), \varphi \geq 0$, and $\phi(x)=1$ for every $\|x\| \leq 1$. Then, for any locally finite Borel measure $\mu$ on $\mathbf{R}^{2}$, we have

$$
\text { L- } \operatorname{supp} \mu=\bigcup_{k=1}^{\infty} \bigcap_{m=1}^{\infty} \bigcup_{n=m}^{\infty}\left\{x \in \mathbf{R}^{2}: \int n^{2} \phi(n(y-x)) d \mu(y) \geq \frac{1}{k}\right\} \text {. }
$$

Note that the above theorem implies that the Lojasiewicz support of a locally finite Borel measure on $\mathbf{R}^{2}$ is measurable.

Theorem 6. The Lojasiewicz support of an extremal dsm has Lebesgue measure 0 .

Proof. Let $\mu$ be an extremal dsm. By Lindenstrauss's result [2], $\mu$ is singular. Since a measure $\nu$ is singular if and only if $D \nu=0$ a.e. with respect to the Lebesgue measure (see Saks, [7], p. 119), we have $D \mu=0$ a.e. Hence, by Theorem $4, d \mu / d \lambda=0$ a.e., and thus L- $\operatorname{supp} \mu$ has Lebesgue measure 0 .

In the next theorem we prove a useful property of the Lojasiewicz support. Note that the theorem fails for the usual support.

Theorem 7. Let $\mu_{1}, \mu_{2}, \ldots$ be a sequence of probability measures on $\mathbf{R}^{2}$, and let $\mu=\sum_{n=1}^{\infty} \alpha_{n} \mu_{n}$, where $\alpha_{1}, \alpha_{2}, \ldots$ is a sequence of positive numbers such that $\sum_{n=1}^{\infty} \alpha_{n}=1$. If

$$
\lambda\left(\mathbf{L}-\operatorname{supp} \mu_{n}\right)=0 \quad \text { for all } n \in \mathbf{N},
$$

then

$$
\lambda(\mathrm{L}-\operatorname{supp} \mu)=0 .
$$

Proof. If $\lambda\left(\mathbf{L}-\operatorname{supp} \mu_{n}\right)=0$, then $\mu_{n}$ is singular for every $n \in \mathbf{N}$. Thus $\mu=$ $\sum_{n=1}^{\infty} \alpha_{n} \mu_{n}$ is singular, and consequently $\lambda(\mathbf{L}-\operatorname{supp} \mu)=0$.

\section{ACKNOWLEDGMENT}

We thank the referee who made suggestions and asked a question which improved the paper.

\section{REFERENCES}

1. P. Antosik, J. Mikusinski and Sikorski, Theory of distributions. The sequential approach, Elsevier-PWN, Amsterdam-Warszawa, 1973.

2. J. Lindenstrauss, A remark on extreme doubly stochastic measures, Amer. Math. Monthly 72 (1965), 379-382.

3. V. Losert, Counter-examples to some conjectures about doubly stochastic measures, Pacific J. Math. 99 (1982), 387-397. 
4. S. Lojasiewicz, Sur la valeur d'une distribution dans un point, Bull. Acad. Pol. Sci. Cl. III 4 (1956), 239-242.

5. $\ldots$, Sur la valeur et le limite d'une distribution dans un point, Studia Math. 16 (1957), 1-36.

6. S. Lojasiewicz, J. Wloka, and Z. Zieleźny, Über eine Definition des wertes einer Distribution, Bull. Acad. Pol. Sci. Cl. III 3 (1955), 479-481.

7. S. Saks, Theory of the integral, Dover Publications, Inc., New York, 1964.

8. Z. Zieleźny, Sur la définition de Lojasiewicz de la valeur d'une distribution dans un point, Bull. Acad. Pol. Sci. Cl. III 3 (1955), 519-520.

Department of Mathematics, University of Central Florida, Orlando, Florida 32816 